\title{
AN EXAMPLE IN THE PROBLEM OF LEAST AREA ${ }^{1}$
}

\author{
WENDELL H. FLEMING
}

1. Introduction. The existence of a surface of least area bounded by a given simple closed curve $C$ (or any finite number of disjoint simple closed curves) in euclidean 3 -space $R^{3}$, and of prescribed finite Euler characteristic and character of orientability is well known (see Courant [1], Douglas [2]; also Radó [5] for the simply connected case). The solution has been generalized by Douglas [3] to include certain prescribed infinite topological types as well. Little is known, however, about the case when the topological type is arbitrary rather than prescribed. In the special case when the boundary curve $C$ has a simply covered orthogonal projection on the perimeter of some convex planar region $B$, the problem for unrestricted topological types has a solution which has a representation in nonparametric form on $B$ and hence is a surface of the type of the disc. This can be proved using a symmetrization argument used by Garabedian and Schiffer $[4$, p. 445] and the existence of a solution of the least area problem for the type of the disc $[1 ; 2 ; 5]$.

The object of the present note is to give an example of a simple closed rectifiable curve $C$ in $R^{3}$ for which the problem of least area with unrestricted topological types has no solution of finite topological type.

The letter $x=\left(x^{1}, x^{2}, x^{3}\right)$ will denote a point in $R^{3} . C, \Gamma, \ldots$ will stand for a closed Fréchet curve in $R^{3}$, nonoriented unless otherwise stated. If the curve is simple closed, the same letter will also be used to denote the point set it occupies. A curve $C$ is termed thin if its set of points is of 2-dimensional Hausdorff measure 0.

Let $V_{1}, \cdots, V_{n}$ be planar 2-cells, each bounded by a rectifiable simple closed curve (in the present applications the boundaries will actually be piecewise analytic). From $V_{1}, \cdots, V_{n}$ we shall define a 2-manifold $D$ with boundary by means of a finite number of identifications of boundary arcs in pairs, after the manner of Siefert-Threlfall [7, Chap. 6]. Identification of a pair of $\operatorname{arcs} \gamma_{1}$ and $\gamma_{2}$ is realized by a homeomorphism of $\gamma_{1}$ onto $\gamma_{2}$. Assume that $D$ is connected and that the boundary $D^{*}$ (union of the unidentified boundary arcs) is a simple closed curve. Let $D^{0}=D-D^{*}$ denote the interior of $D$. By $[7, \mathrm{p} .142]$,

Presented to the Society, May 1, 1954; received by the editors August 19, 1954 and, in revised form, December 13, 1954 and October 24, 1955.

1 This paper was sponsored by the Office of Ordnance Research, U. S. Army. 
$D$ is topologically a disc with a finite number $h$ handles (orientable case) or $q$ cross-caps (nonorientable case). The topological type $\tau$ of $D$ is specified by its orientability or nonorientability and characteristic $r$, where $r=2 h-1$ or $q-1$ according as $D$ is orientable or nonorientable.

Let $w$ denote a point of $D$, and $x(w)$ a continuous mapping from $D$ to $R^{3}$ with the following property:

(1.1) For each $i=1, \cdots, n, x(w)$ agrees in $V_{i}$ with a mapping $x_{i}(u, v)$ continuous on $V_{i}$ and satisfying a Lipschitz condition on each closed subset of $V_{i}^{0}$.

Let

$$
\begin{aligned}
& a(x, D)=\sum_{i=1}^{n} \iint_{V_{i}^{0}}\left[E G-F^{2}\right]^{1 / 2} d u d v, \\
& I(x, D)=\sum_{i=1}^{n} \iint_{V_{i}^{0}} \frac{1}{2}(E+G) d u d v
\end{aligned}
$$

denote, respectively, the classical area integral and the Dirichlet integral, where $E, F$, and $G$ have their usual meaning in differential geometry. The quantities $a(x, D)$ and $I(x, D)$ are invariant under subdivision and conformal transformation of the cells $V_{i}$.

If $V_{1}, \cdots, V_{n}$ are triangles and $x_{i}(u, v)$ is one-one and linear on each $V_{i}$, then $x(w)$ defines a (Fréchet) polyhedron $P$ of the same type as $D$. On $D^{*}, x(w)$ represents a closed polygonal curve $C$, termed the boundary of $P$. Let $e(P)$ denote the elementary area of $P$. For such a piecewise linear representation $x(w)$ of $P, e(P)=a(x, D)$.

Given a simple closed curve $C$ in $R^{3}$ and $\tau$, let

$$
m(C, \tau)=\inf _{\left\{P_{n}\right\}} \lim _{n} \inf e\left(P_{n}\right),
$$

where $P_{n}$ is a polyhedron of type $\tau, n=1,2, \cdots$, whose boundary $C_{n}$ tends to $C$ in the Fréchet sense. $m(C, \tau)$ is termed the least area for type $\tau$ and boundary $C$, and agrees with the inf of the Lebesgue area for surfaces of type $\tau$ and boundary $C$ [2, pp. 295-296]. By least area for unrestricted topological type and boundary $C$ we mean

$$
m(C)=\inf _{\left\{P_{n}\right\}} \lim _{n} \inf e\left(P_{n}\right),
$$

where as before the boundary $C_{n}$ of $P_{n}$ tends to $C$ but now the type of $P_{n}$ is arbitrary.

(1.2) Lemma. Let $C$ be a closed, rectifiable Fréchet curve in $R^{3}$. Let $x(w)$ be a continuous mapping from a domain $D$ of type $\tau$, defined as 
above by planar 2-cells $V_{1}, \cdots, V_{n}$, such that:

(a) (1.1) holds;

(b) $I(x, D)$ is finite;

(c) $x(w)$ represents $C$ on $D^{*}$;

(d) for each $i=1, \cdots, n$, the mapping $x_{i}(u, v)$ agreeing with $x(w)$ in $V_{i}$ represents a thin curve on $V_{i}^{*}$.

Then there exists a sequence $P_{n}$ of polyhedra of type $\tau$ whose boundaries $C_{n}$ tend to $C$ in the Fréchet sense such that $\lim _{n} e\left(P_{n}\right)=a(x, D)$.

By making conformal transformations, we may suppose all $V_{i}$ are polygons. Lemma (1.2) is then essentially a special case of approximation theorems recently published by Young [8] in his treatment of the general problem of minimum for parametric surfaces of prescribed, finite topological type (see especially [8, (2.3), (3.2), (4.2), (6.5) ]). Young's methods actually yield a more general form of (1.2), obtained by replacing pointwise identification along identified boundary arcs by a less restrictive type of boundary identification.

We shall need the fundamental existence theorem $[1 ; 2]$ for the problem of least area with prescribed type $\tau$, which is quoted as (1.3) below. The domain $D$ appearing in the solution is of a particular normal form, and is defined somewhat differently than above. For definiteness we shall follow Courant [1], where the normal domain is a half-plane domain with a certain finite set of identified slits parallel to the real axis. To represent a half-plane slit domain in the form above, defined by a finite number of planar 2-cells $V_{1}, \cdots, V_{n}$, first transform $D$ into a bounded domain by inverting about a point in the complementary half-plane, and then make a suitable dissection. Given a boundary point $w^{*} \in D^{*}$, we may further arrange (by means of elementary conformal transformations, reflections, subdivision, and combining of cells) that $V_{1}$ is the upper half $K$ of the unit circle and that $w^{*}$ corresponds to the point $(0,0)$ of $K$.

For such normal domains, $D^{0}$ has an analytic as well as a topological structure. In particular, harmonic functions can be defined in $D^{0}\left(x(w)\right.$ is harmonic in $D^{0}$ if near each point $w^{0} \in D^{0}$ the components $x^{j}(w), j=1,2,3$, are harmonic when referred to a set of local variables $(u, v)[1$, p. 76]).

(1.3) Let $C$ be a simple closed curve in $R^{3}$ such that $m(C, \tau)$ is finite, and either: (i) $\tau$ is the type of the disc, or (ii) $\tau$ is some higher type and $m(C, \tau)<m\left(C, \tau^{\prime}\right)$ for any $\tau^{\prime}$ of characteristic smaller than that of $\tau$. Then there exists a normal domain $D$ of type $\tau$ and $x(w)$ continuous on $D$, such that:

(a) $x(w)$ is harmonic in $D^{0}$;

(b) $E=G, F=0$ in $D^{0}$; 
(c) $a(x, D)=I(x, D)=m(C, \tau)$;

(d) $x$ (w) maps $D^{*}$ one-one onto $C$.

We shall consider here only rectifiable curves $C$, for which the hypothesis $m(C, \tau)$ finite holds for any $\tau$. Items (1.3a) and (1.3b) express that $x(w)$ is a minimal surface.

2. The example. We begin with an example due to Douglas $\left[2\right.$, p. 209] of a simple closed curve $\Gamma_{0}$ for which the minimum area for surfaces of the type of the disc is much greater than the absolute minimum area. The equations

$$
\begin{aligned}
& x^{1}=-1+\cos \theta(2-\cos \phi), \\
& x^{2}=\sin \theta(2-\cos \phi), \\
& x^{8}=\sin \phi,
\end{aligned}
$$$$
-\pi \leqq \theta, \phi \leqq \pi,
$$

define a torus $T$ with center $(-1,0,0)$, major radius 2 , and minor radius 1 . Let us denote this mapping by $f(\theta, \phi)$. For given fixed $\delta$, $0<\delta<2^{-5}$, let

$$
S_{1}=\left[(\theta, \phi)|| \theta \mid \leqq \frac{\delta}{2}\right], \quad S_{2}=\left[(\theta, \phi)|| \phi \mid \leqq \frac{\delta}{2}\right] .
$$

Let $S$ denote the domain obtained from $S_{1} \cup S_{2}$ upon identifying $(-\pi, \phi)$ with $(\pi, \phi)$ and $(\theta,-\pi)$ with $(\theta, \pi) . S$ is of the type of the disc with one handle, and $f(\theta, \phi)$ maps $S$ onto a portion of $T$ of area $<7 \pi \delta$. The image of $S^{*}$ is a simple closed curve, which we denote by $\Gamma_{0}$. For simply connected surfaces bounded by $\Gamma_{0}$, the area is at least $2 \pi(1-\delta)^{2}$. This is a special case of $(2.1)$ below.

For each pair of real numbers $\rho_{1}, \rho_{2}, \rho_{1}<\rho_{2}$, let

$$
B\left(\rho_{1}, \rho_{2}\right)=\left[x \in R^{3} \mid \rho_{1}<x^{1}<\rho_{2}\right] .
$$

(2.1) Leмma. Let $\Gamma$ be a simple closed curve in $R^{3}$ such that $\Gamma \cap B(-2+\delta, 2-\delta)=\Gamma_{0} \cap B(-2+\delta, 2-\delta)$. Let $D$ be $a$ planar 2-cell bounded by a simple closed curve and $x(u, v)$ a mapping from $D$ to $R^{3}$ such that:

(a) $x(u, v)$ is continuous on $D$ and Lipschitzian on each closed subset of $D^{0}$;

(b) $a(x, D)$ is finite;

(c) On $D^{*}, x(u, v)$ represents $\Gamma$.

Then $a(x, D) \geqq 2 \pi(1-\delta)^{2}$.

Proof. We may suppose $D$ a square. Let $0<\epsilon<\delta$. By a known theorem there exists a piecewise linear mapping $y(u, v)$ such that $|y(u, v)-x(u, v)|<\epsilon$ for $(u, v) \in D$ and $a(y, D)<a(x, D)+\epsilon$. Let 
$\left(u_{1}, v_{1}\right), \cdots,\left(u_{4}, v_{4}\right)$ be four points of $D^{*}$ such that $x^{1}\left(u_{i}, v_{i}\right)=(-1)^{i+1}$, $i=1,2,3,4$, and $\left(u_{1}, v_{1}\right) \cup\left(u_{3}, v_{3}\right)$ separates $\left(u_{2}, v_{2}\right)$ and $\left(u_{4}, v_{4}\right)$ in $D^{*}$. Let

$$
K=\left[(u, v) \in D \mid y^{1}(u, v)=0\right] .
$$

Either $K$ separates $\left(u_{1}, v_{1}\right)$ and $\left(u_{3}, v_{3}\right)$ in $D$, or $K$ separates $\left(u_{2}, v_{2}\right)$ and $\left(u_{4}, v_{4}\right)$ in $D$. For if not, then $\left(u_{1}, v_{1}\right),\left(u_{3}, v_{3}\right)$ can be joined in $D-K$ by a polygonal arc $\pi_{1}$, and $\left(u_{2}, v_{2}\right),\left(u_{4}, v_{4}\right)$ can be joined in $D-K$ by a polygonal $\operatorname{arc} \pi_{2} . \pi_{1} \cap \pi_{2}$ is nonempty since $\left(u_{1}, v_{1}\right) \cup\left(u_{3}, v_{3}\right)$ separates $\left(u_{2}, v_{2}\right)$ and $\left(u_{4}, v_{4}\right)$ in $D^{*}$. However, $y^{1}(u, v)>0$ on $\pi_{1}$ and $\boldsymbol{y}^{1}(u, v)<0$ on $\pi_{2}$, a contradiction.

For definiteness, suppose $\left(u_{1}, v_{1}\right)$ and $\left(u_{3}, v_{3}\right)$ are separated by $K$, the remaining case being similar. Since $y(u, v)$ is piecewise linear, $\left(u_{1}, v_{1}\right)$ and $\left(u_{3}, v_{3}\right)$ are then separated by a polygonal arc $\pi \subset K$. We may suppose $\pi \cap D^{*}$ consists of only two points. Then $D^{*}-\pi$ consists of two disjoint arcs $\gamma_{1}, \gamma_{2}$ with $\left(u_{1}, v_{1}\right) \in \gamma_{1},\left(u_{3}, v_{3}\right) \in \gamma_{2}$. On $\gamma_{1}, \gamma_{2}$, respectively, $x(u, v)$ represents nonoverlapping arcs $\Gamma^{\prime}, \Gamma^{\prime \prime}$ of $\Gamma$ whose endpoints are situated in $B(-\delta, \delta)$ since $\epsilon<\delta$. If we suppose for definiteness that $x^{2}\left(u_{1}, v_{1}\right)>0$, then

$$
\begin{aligned}
\Gamma^{\prime} \cap B(\delta, 2-\delta) & =\left[x \in \Gamma \cap B(\delta, 2-\delta) \mid x^{2}>0\right], \\
\Gamma^{\prime \prime} \cap B(\delta, 2-\delta) & =\left[x \in \Gamma \cap B(\delta, 2-\delta) \mid x^{2}<0\right] .
\end{aligned}
$$

If $Y(p)$ is a continuous mapping of an oriented simple closed planar curve $\zeta$ into a plane $A$, let $\mu(q, Y, \zeta)$ denote as in [6] the topological index with respect to a point $q \in A$; for any arc $\eta$ of $\zeta$ on which $Y(p) \neq q$, let $V_{\eta}[\arg (Y(p)-q)]$ denote the variation in argument of $Y(p)-q$ on $\eta$. The following known properties hold (cf. [6, esp. pp. 142-149]):

(1) Let $\zeta=\eta_{1} \cup \ldots \cup \eta_{n}$, where $\eta_{1}, \cdots, \eta_{n}$ are pairwise nonoverlapping arcs. If $Y(p) \neq q$ on $\zeta$, then

$$
\mu(q, Y, \zeta)=\frac{1}{2 \pi} \sum_{i=1}^{n} V_{\eta_{i}}[\arg (Y(p)-q)] .
$$

(2) Let $l$ be a straight line not containing $q$. Let $Y(p)$ be in the closed half-space bounded by $l$ not containing $q$, for all $p \in \eta$, and be on $l$ at the end points of $\eta$. Then $V_{\eta}[\arg (Y(p)-q)]$ equals the angle $Y\left(p_{1}\right) q Y\left(p_{2}\right)$, where $p_{1}$ is the initial end point of $\eta$ and $p_{2}$ the final.

(3) $\mu\left(q, Y_{1}, \zeta\right)=\mu\left(q, Y_{2}, \zeta\right)$, if

$$
\sup _{\nu \in \zeta}\left|Y_{1}(p)-Y_{2}(p)\right|<\inf _{p \in S}\left|Y_{1}(p)-q\right| .
$$


(4) If $D$ is a planar 2-cell, $Y(p)$ is defined and continuous on $D$, and $\mu\left(q, Y, D^{*}\right) \neq 0$, then $q \in Y(D)$.

Let $D_{1}$ denote the 2 -cell bounded by $\gamma_{1} \cup \pi$. Let $X(u, v), Y(u, v)$ denote the plane mappings obtained from $x(u, v), y(u, v)$, respectively, upon setting the second coordinate $x^{2}$ equal to 0 . Clearly, $\mid X(u, v)$ $-Y(u, v) \mid<\epsilon,(u, v) \in D$.

Let $\Gamma^{\wedge}$ denote the ellipse

$$
x^{1}=-1+\cos \frac{\delta}{2}(2-\cos \phi), \quad x^{2}=0, \quad x^{3}=\sin \phi, \quad-\pi \leqq \phi \leqq \pi .
$$

The circular disc $\left(x^{1}-1\right)^{2}+\left(x^{3}\right)^{2} \leqq(1-\delta)^{2}$ in the plane $x^{2}=0$ is inside $\Gamma^{-} . X(u, v)$ represents the part of $\Gamma^{-}$for which $\delta<x^{1}<2-\delta$ on a pair of open arcs of $D_{1}^{*}$ with disjoint closures, and $X^{1}(u, v)$ is outside this interval on the complementary pair of arcs of $D_{1}^{*}$. If $q=\left(x^{1}, x^{8}\right)$ and $\left(x^{1}-1\right)^{2}+\left(x^{8}\right)^{2}<(1-\delta)^{2}$, then $\left|\mu\left(q, X, D_{1}^{*}\right)\right|=1$ by (1) and (2) (let us assign a definite orientation to $D_{1}^{*}$ as required in the definition of index $\mu$ ). If $\left(x^{1}-1\right)^{2}+\left(x^{3}\right)^{2}<(1-\delta-\epsilon)^{2}$, then $\left|\mu\left(q, Y, D_{1}^{*}\right)\right|=1$ by (3). By (4) and the fact that $Y(u, v)$ is piecewise linear,

$$
a\left(Y, D_{1}\right) \geqq \pi(1-\delta-\epsilon)^{2} .
$$

Applying a similar argument to the 2-cell $D_{2}$ bounded by $\gamma_{2} \cup \pi$, $a\left(Y, D_{2}\right) \geqq \pi(1-\delta-\epsilon)^{2}$. Since $Y$ is the projection of $y$, and $y$ is piecewise linear,

$a(y, D)=a\left(y, D_{1}\right)+a\left(y, D_{2}\right) \geqq a\left(Y, D_{1}\right)+a\left(Y, D_{2}\right) \geqq 2 \pi(1-\delta-\epsilon)^{2}$.

Since $\epsilon$ is arbitrary, this proves (2.1).

Let $U_{k}(x)$ denote the similarity transformation defined by

$$
\begin{aligned}
U_{k}(x) & =2^{-k} x+a_{k}, \\
a_{k} & =\left[2 \delta^{-1}\left(1-2^{-k}\right), 0,0\right], \quad k=0,1,2, \cdots .
\end{aligned}
$$

Let $\Gamma_{k}=U_{k}\left(\Gamma_{0}\right)$; the curves $\Gamma_{k}$ shrink down to a point $x^{*}=\left(2 \delta^{-1}, 0,0\right)$. Let $\alpha_{0}$ denote an open arc of $\Gamma_{0}$ of length $<\pi \delta^{2} / 4$ and midpoint

$$
f\left(\frac{\delta}{2}, \pi\right)=\left(-1+3 \cos \frac{\delta}{2}, 3 \sin \frac{\delta}{2}, 0\right) .
$$

Let $\beta_{0}$ denote an open arc of $\Gamma_{1}$ of length $<\pi \delta^{2} / 4$ with midpoint

$$
U_{1}\left[f\left(\pi, \frac{\delta}{2}\right)\right]=\left(-\frac{3}{2}+\frac{1}{2} \cos \frac{\delta}{2}+\delta^{-1}, 0, \frac{1}{2} \sin \frac{\delta}{2}\right) .
$$

Let $d_{0}$ and $e_{0}$ denote a pair of line segments whose left (right) end 
points are the ends of $\alpha_{0}\left(\beta_{0}\right) . d_{0} \cap e_{0}$ is empty. Let

$$
C=\left\{x^{*}\right\} \cup\left[\bigcup_{k=0}^{\infty} U_{k}\left(\Gamma_{0} \cup d_{0} \cup e_{0}\right)-\bigcup_{k=0}^{\infty} U_{k}\left(\alpha_{0} \cup \beta_{0}\right)\right] \text {. }
$$

$C$ is a rectifiable simple closed curve, and has the desired property:

(2.3) Theorem. For the rectifiable simple closed curve $C$ defined by (2.2), $m(C, \tau)>m(C)$ for every finite topological type $\tau$ (no surface of finite type provides least area for unrestricted types).

REMARK. The only really essential property of $\Gamma_{0}$ used in constructing $C$ is that the absolute minimum area for $\Gamma_{0}$ is much less than the minimum area for surfaces of the type of the disc bounded by $\Gamma_{0}$. As pointed out by R. H. Bing, there exists a simple closed curve $\Gamma$ with this property situated in the union of two planes. For example, let $\Gamma=\Sigma_{1} \cup \ldots \cup \Sigma_{5}$, where

$$
\begin{aligned}
& \Sigma_{1}=\left[x \mid\left(x^{1}\right)^{2}+\left(x^{3}\right)^{2}=1, x^{3} \geqq 0, x^{2}=0\right], \\
& \Sigma_{2}=\left[x \mid\left(x^{1}\right)^{2}+\left(x^{3}\right)^{2}=(1-\delta)^{2}, x^{8} \geqq 0, x^{2}=0\right], \\
& \Sigma_{3}=\left[x \mid\left(x^{1}+\delta / 2\right)^{2}+\left(x^{2}\right)^{2}=(1-\delta / 2)^{2}, x^{2} \geqq 0, x^{8}=0\right], \\
& \Sigma_{4}=\left[x \mid\left(x^{1}+\delta / 2\right)^{2}+\left(x^{2}\right)^{2}=(1-3 \delta / 2)^{2}, x^{2} \geqq 0, x^{8}=0\right], \\
& \Sigma_{5}=\left[x \mid\left(x^{1}-1+\delta\right)^{2}+\left(x^{2}\right)^{2}=\delta^{2}, x^{2} \leqq 0, x^{3}=0\right] .
\end{aligned}
$$

By a construction similar to the one above, we obtain a simple closed rectifiable curve $C$ situated in the union of the planes $x^{2}=0$ and $x^{3}=0$ for which (2.3) holds. Professor Bing also suggested certain important simplifications in an earlier version of the proof of (2.1).

Proof of (2.3). We prove (2.3) by contradiction. Since $C$ is rectifiable, $m(C)$ is finite; moreover, by definition, $m(C, \tau) \geqq m(C)$ for all $\tau$. Suppose $m(C, \tau)=m(C)$ for some finite type $\tau$, in particular for some $\tau_{0}$ of smallest characteristic. Then $C$ and $\tau_{0}$ satisfy the hypotheses of (1.3); therefore, there exist a normal domain $D$ of type $\tau_{0}$ and $x(w)$ defined in $D$ such that (1.3a)-(1.3d) hold.

Let us first note some geometric properties satisfied by $C$. Let

$$
\begin{aligned}
& b_{k}=a_{k}^{1}+2^{-k+1}, \\
& b_{k}^{\prime}=a_{k+1}^{1}-(1+\delta) 2^{-k}, \quad k=0,1, \cdots .
\end{aligned}
$$

For $b_{k} \leqq s \leqq b_{k}{ }^{\prime}$, the plane $x^{1}=s$ separates $\Gamma_{k}$ and $\Gamma_{k+1}$, and meets $C$ in a pair of points $x_{s}, \bar{x}_{8}$ interior to the line segments $d_{k}=U_{k}\left(d_{0}\right)$, $e_{k}=U_{k}\left(e_{0}\right)$, respectively.

Let $L$ denote the solid right circular cone in $R^{3}$ with vertex $x^{*}$, defined by 


$$
\left[\left(x^{2}\right)^{2}+\left(x^{8}\right)^{2}\right]^{1 / 2} \leqq \frac{\delta}{2} \frac{2 \delta^{-1}-x^{1}}{1-\delta}, x^{1} \leqq 2 \delta^{-1} .
$$

We show that $C \subset L$; to do this it will suffice to show that all $\Gamma_{k} \subset L$. Consider the case $k=0$ and set $\theta=\delta / 2$ in the definition of $\Gamma_{0}$. It suffices to consider $\pi / 2 \leqq \phi \leqq 3 \pi / 4$, for which values of $\phi, \sin ^{2} \phi \geqq 1 / 2$. Then

$$
\begin{aligned}
\left(x^{2}\right)^{2}+\left(x^{3}\right)^{2} & =\sin ^{2} \frac{\delta}{2}(2-\cos \phi)^{2}+\sin ^{2} \phi \\
& \leqq \sin ^{2} \phi\left(1+\frac{9}{2} \delta^{2}\right) .
\end{aligned}
$$

Let $\rho=\delta(1-\delta)^{-1 / 2} / 2$. Then in a $(\xi, \eta)$ plane, the circle $(\xi-1)^{2}+\eta^{2}=1$ is tangent to the line $\eta=\rho\left(2 \delta^{-1}-\xi\right)$. Setting $\xi=1-\cos \phi, \eta=\sin \phi$, then $\sin \phi \leqq \rho\left[2 \delta^{-1}-(1-\cos \phi)\right]$, whence

$$
\left[\left(x^{2}\right)^{2}+\left(x^{3}\right)^{2}\right]^{1 / 2} \leqq \rho\left(1+\frac{9}{2} \delta^{2}\right)^{1 / 2}\left[2 \delta^{-1}-(1-\cos \phi)\right] .
$$

Since $1-\cos \phi \geqq-1+(\cos \delta / 2)(2-\cos \phi)=x^{1}$, and $\left(1+9 \delta^{2} / 2\right)^{1 / 2}$ $\cdot(1-\delta)^{1 / 2} \leqq 1$ for $0<\delta<2^{-5}$,

$$
\left[\left(x^{2}\right)^{2}+\left(x^{3}\right)^{2}\right]^{1 / 2} \leqq \frac{\delta}{2} \frac{2 \delta^{-1}-x^{1}}{1-\delta} .
$$

Thus $x=\left(x^{1}, x^{2}, x^{3}\right) \in L$. The cases $\theta=-\delta / 2, \phi= \pm \delta / 2$, and $k=1,2, \cdots$ are similar. Thus, $C \subset L$. Since $L$ is a closed convex region containing $C$ and $x(w)$ is harmonic in $D^{0}$, the values $x(w)$ must lie in $L$ for all $w \in D$ by the maximum modulus principle for harmonic functions.

For each $s$, the intersection of $L$ with the plane $x^{1}=s$ is a circular disc whose radius we denote by $r_{s}$. One verifies that for $s=b_{k}{ }^{\prime}$, $r_{s}<2^{-k-1}(1+3 \delta), k=0,1, \cdots$.

By $(1.3 \mathrm{~d}), x^{*}=x\left(w^{*}\right)$ for exactly one $w^{*} \in D^{*}$. Let $D$ be represented by 2-cells $V_{1}, \cdots, V_{n}$ as in $\$ 1$. By (1.3a), the image of each boundary $V_{i}^{*}$ is a finite or countable sum of rectifiable arcs and points, and hence is thin. By a previous remark, we may suppose that $V_{1}$ is the upper half circle $K$ and that $w^{*}$ corresponds to $(0,0)$. The function $x_{1}(u, v)$ is continuous in $K$; in $K^{0}, x_{1}(u, v)$ is a minimal surface. For brevity, let us drop the subscript 1 . Let $\lambda$ denote the straight portion of $K^{*}$. By the reflection principle [1, p. 119], $x(u, v)$ has an analytic extension as a minimal surface across any arc of $\lambda$ which maps onto an arc interior to some segment $d_{k}$ or $e_{k}$ of $C$. 
By construction of $C$ and the maximum modulus principle, $x^{1}(w)$ $<2 \delta^{-1}$ for $w \neq w^{*}$. Hence, $x^{1}(u, v)$ has an upper bound $s_{0}<2 \delta^{-1}$ on $K^{*}-\lambda$. Let $k_{0}$ be such that $s_{0}<b_{k_{0}}$; for $b_{k} \leqq s \leqq b_{k}{ }^{\prime}, k>k_{0}$, let

$$
W_{\mathbf{s}}=\left[(u, v) \in K \mid x^{1}(u, v)=s\right] .
$$

$W_{s} \cap K^{*}$ consists of two points $\left(u_{s}, 0\right),\left(\bar{u}_{s}, 0\right)$ in $\lambda$, with $x_{s}=x\left(u_{s}, 0\right)$, $\bar{x}_{s}=x\left(\bar{u}_{s}, 0\right)$.

Let $K^{\wedge}$ denote $K^{0}$ plus the union of all open arcs of $\lambda$ across which $x(u, v)$ has an analytic extension. $W_{s} \subset K^{-}$for $s \in\left(b_{k}, b_{k}^{\prime}\right), k>k_{0}$. Since $x^{1}(u, v)$ is harmonic and not constant, the equation $x_{u}^{1}=x_{v}^{1}=0$ has only isolated solutions in $K^{\curlywedge}$. Moreover, for all $(u, v)$,

$$
E=\frac{1}{2}(E+G) \geqq \frac{1}{2}\left[\left(x_{u}^{1}\right)^{2}+\left(x_{v}^{1}\right)^{2}\right] .
$$

Suppose $\left(x_{u}^{1}\right)^{2}+\left(x_{v}^{1}\right)^{2}>0$ on $W_{8}$. Then $W_{8}$ is an analytic arc; i.e., $W_{\text {s }}$ has a representation $u=u(t), v=v(t), 0 \leqq t \leqq 1$, which is one-one and analytic from $(0,1)$ to $W_{s}$ such that $\left(u^{\prime}\right)^{2}+\left(v^{\prime}\right)^{2}>0$. Since $x^{2}(u, v)$ and $x^{3}(u, v)$ are analytic in $(u, v), \phi(t)=x^{2}[u(t), v(t)]$ and $\psi(t)=x^{3}[u(t), v(t)]$ are analytic; by an easy calculation

$$
\left(\phi^{\prime}\right)^{2}+\left(\psi^{\prime}\right)^{2}=E\left[\left(u^{\prime}\right)^{2}+\left(v^{\prime}\right)^{2}\right]>0 .
$$

Thus, the image of $W_{s}$ is likewise an analytic arc, provided $x(u, v)$ is one-one on $W_{\text {s. }}$.

I assert that for each $k>k_{0}, x(u, v)$ is one-one on $W_{8}$ for a dense set of $s \in\left(b_{k}, b_{k}^{\prime}\right)$. If this fails to hold for some $k$, then there is a subinterval $\Delta$ of $\left(b_{k}, b_{k}^{\prime}\right)$ such that, for $s \in \Delta,\left(x_{u}^{1}\right)^{2}+\left(x_{v}^{1}\right)^{2}>0$ on $W_{s}$ and the equation $x(u, v)=x(\bar{u}, \bar{v})$ has a solution for distinct points $(u, v)$, $(\bar{u}, \bar{v})$ of $W_{\mathrm{s}}$. Since $x(u, v)$ is analytic in $(u, v)$, it then follows from a known theorem that distinct analytic arcs $\gamma_{1}$ and $\gamma_{2}$ in $K^{\wedge}$ exist on which $x(u, v)$ describes the same curve. This contradicts a remark of Courant $[1$, p. 159] that a minimal surface providing absolute least area can have no line of self-intersection. ${ }^{2}$

Thus, for each $k>k_{0}$, there is a dense subset $J_{k}$ of $\left(b_{k}, b_{k}^{\prime}\right)$ for which $W_{8}$ and its image are analytic arcs. Let us now fix $k>k_{0}+1$ and $s \in J_{k-1}$ close enough to $b_{k-1}^{\prime}$ that $r_{s}<2^{-k}(1+3 \delta)$. Let $l$ denote the line segment joining $x_{s}$ and $\bar{x}_{\mathrm{s}}$ and $\gamma$ the analytic arc $x\left(W_{\mathrm{s}}\right)$. We exclude

2 The form of Courant's remark we want here is the following: Let $x(w)$ satisfy (1.3a)-(1.3d) relative to the normal domain $D$ and simple closed curve $C$. Let there exist two analytic arcs $\gamma_{1}$ and $\gamma_{2}$ situated in $D^{0}$, or $D^{0}$ plus an arc of $D^{*}$ across which $x(w)$ has an analytic extension, on which $E \neq 0$ and $x(w)$ describes the same curve. Then $a(x, D)>m(C)$. Courant gives only a heuristic proof of the remark; however the details are elementary. 
the case $l=\boldsymbol{\gamma}$, which can be dealt with by a simplification of the argument below. Since $l$ and $\gamma$ are analytic arcs, $l \cap \gamma$ is a finite set of points $x_{0}, x_{1}, \cdots, x_{n}$, ordered from $x_{8}$ to $\bar{x}_{8}$ following the order of $l$, with $x_{0}=x_{s}, x_{n}=\bar{x}_{s}$.

Let $H$ denote the plane $x^{1}=s$. The components of $H-(l \cup \gamma)$ can be colored "black" and "white" in such a way that any two components with a common boundary arc have opposite colors. The proof of this is elementary, and will merely be sketched. First, it suffices to replace $\gamma$ by a suitable polygonal arc $\gamma_{1}$ likewise meeting $l$ in $x_{0}, \cdots, x_{n}$ and precisely those points. Next, we may eliminate the case where $\gamma_{1}$ touches $l$ at some $x_{i}, 1 \leqq i \leqq n-1$, but does not cross $l$ at $x_{i}$, by a suitable slight deformation of $\gamma_{1}$ near $x_{i}$. We then proceed by induction on $n$. The cases $n=1,2$ are trivial. For $n>2$ it must be true that for some $i, 1 \leqq i \leqq n-2, x_{i}$ and $x_{i+1}$ are joined by an arc $\zeta$ of $\gamma_{1}$ which does not meet $l$ between its end points. Let $\zeta^{\prime}$ denote a suitable slightly larger arc of $\gamma_{1}$, obtained by extending $\zeta$ past $x_{i}$ and $x_{i+1}$ along $\gamma_{1}$. Replace $\zeta^{\prime}$ by a line segment joining its end points. There are now $n-2$ intersections; hence, we can color. From this coloring it is easy to define a coloring for the original configuration.

For definiteness, color the unbounded component white. Let $Z_{1}, \cdots, Z_{\mu}$ denote the black components plus their boundaries. Each $Z_{i}$ is a 2 -cell bounded by a finite number of analytic arcs, and each point of $\cup_{\gamma}$ is in at least one $Z_{i}^{*} . Z_{1} \cup \ldots \cup Z_{\mu} \subset H \cap L$, a circle of radius $r_{\mathbf{s}}$.

Let us now replace $k$ by $k+1$ and define $s^{\prime}, l^{\prime}, \gamma^{\prime}, Z_{1}^{\prime}, \cdots, Z_{\nu}^{\prime}$ correspondingly. We have upon letting $X_{i}, X_{j}^{\prime}$ denote identity maps:

$$
\begin{aligned}
& \sum_{i=1}^{\mu} a\left(X_{i}, Z_{i}\right) \leqq \pi r_{i}^{2}<\pi 2^{-2 k}(1+3 \delta)^{2}, \\
& \sum_{j=1}^{p} a\left(X_{i}^{\prime}, Z_{i}^{\prime}\right) \leqq \pi r_{s^{\prime}}^{2}<\pi 2^{-2(k+1)}(1+3 \delta)^{2}=\pi 2^{-2 k} \frac{(1+3 \delta)^{2}}{4} .
\end{aligned}
$$

Let $f_{k}=U_{k} f$ (cf. definition of the torus $T$ ).

$$
a\left(f_{k}, S\right)<\pi 2^{-2 k}(7 \delta) .
$$

In addition, by construction of $C$, there exist Lipschitzian one-one mappings $g(u, v), g^{\prime}(u, v)$ from squares $Z, Z^{\prime}$, respectively, such that: (1) on $Z^{*}, g$ represents the curve made up of $\beta_{k-1}, l$, and the parts of $d_{k-1}$ and $e_{k-1}$ with $x^{1}>s$, while $g^{\prime}$ represents on $\left(Z^{\prime}\right)^{*}$ the curve made up of $\alpha_{k}, l^{\prime}$, and the parts of $d_{k}$ and $e_{k}$ with $x^{1}<s^{\prime}$; (2) $a(g, Z)$ $<\pi 2^{-2(k-1)}(\delta / 4), a\left(g^{\prime}, Z^{\prime}\right)<\pi 2^{-2 k}(\delta / 4)$.

Let $A$ denote the component of $K-W_{8}$ containing $(0,0), A^{\prime}$ being 
defined similarly when $s^{\prime}$ replaces $s$. Since $s^{\prime}>s, A^{\prime} \subset A . Q=\left(A-A^{\prime}\right)$ $\cup W_{\text {s }}$ is a 2-cell. On $Q^{*}, x(u, v)$ describes a simple closed curve $C_{1}$ whose image under the similarity transformation $U_{k}^{-1}$ satisfies the hypotheses of (2.1). Hence, by (2.1),

$$
a(x, Q) \geqq \pi 2^{-2 k}\left[2(1-\delta)^{2}\right] .
$$

Consider the following collection: (1) $D-Q^{0}{ }^{3}$ (2) $Z_{1}, \cdots, Z_{\mu}$; (3) $Z_{1}^{\prime}, \cdots, Z_{v}^{\prime}$; (4) $S$; (5) $Z$; (6) $Z^{\prime}$. For $i=1, \cdots, \mu$, let $p \in Z_{i}^{*}$ be identified with $x^{-1}(p) \in W_{s}$ if $p \in \gamma$ and is not an isolated point of $\gamma \cap Z_{i}^{*}$, and with $g^{-1}(p) \in Z^{*}$ otherwise (similar boundary identifications for $\left.Z_{j}^{\prime}, j=1, \cdots, \nu\right)$. In addition, identify each pair of points of $S^{*}$ and $Z^{*}\left(S^{*}\right.$ and $\left.\left(Z^{\prime}\right)^{*}\right)$ corresponding to the same point of $\beta_{k-1}\left(\alpha_{k}\right)$. Let $D_{1}$ denote the domain obtained from (1)-(6) under these identifications, and $x_{1}(w)$ the mapping from $D_{1}$ which agrees with $x$, $X_{i}, X_{j}^{\prime}, f_{k}, g$, and $g^{\prime}$ in their respective domains of definition.

$D_{1}$ is a domain of the kind defined in $\$ 1$. To see this we need only verify that each identified arc is identified with just one other, $D$ is connected, and the unidentified boundary arcs form a simple closed curve (cf. [7]). Moreover, $x_{1}(w)$ and $C$ satisfy the hypotheses of (1.2) on $D_{1}$.

Now $a\left(x, D-Q^{0}\right)=a(x, D)-a(x, Q)=m(C)-a(x, Q)$. Hence, recalling that $0<\delta<2^{-5}$,

$$
\begin{aligned}
a\left(x_{1}, D_{1}\right)= & a\left(x, D-Q^{0}\right)+\sum_{i=1}^{\mu} a\left(X_{i}, Z_{i}\right)+\sum_{j=1}^{\prime} a\left(X_{j}^{\prime}, Z_{j}^{\prime}\right) \\
& +a\left(f_{k}, S\right)+a(g, Z)+a\left(g^{\prime}, Z^{\prime}\right) \\
\leqq & m(C)+\pi 2^{-2 k}\left[-2(1-\delta)^{2}+\frac{5}{4}(1+3 \delta)^{2}+7 \delta+\frac{5 \delta}{4}\right] \\
= & m(C)+\frac{\pi}{4} 2^{-2 k}\left[-3+79 \delta+37 \delta^{2}\right]=m(C)+\sigma,
\end{aligned}
$$

where $\sigma<0$. By (1.2), there exists a polyhedron $P$ with boundary arbitrarily close to $C$ such that $e(P)<m(C)+\sigma / 2$, contradicting the definition of $m(C)$. This proves (2.3).

\section{REFERENCES}

1. R. Courant, Dirichlet's principle, conformal mapping, and minimal surfaces, Interscience, 1950.

2. J. Douglas, Minimal surfaces of higher topological structure, Ann. of Math. vol. 40 (1939) pp. 205-298.

I.e., $V_{1}-Q^{0}, V_{2}, \cdots, V_{n}$. 
3. - Green's function and the problem of Plateau. The most general form of the problem of Plateau, Amer. J. Math. vol. 61 (1939) pp. 545-608.

4. P. Garabedian, and M. Schiffer, On a double integral variational problem, Canadian Journal of Mathematics vol. 6 (1954) pp. 441-446.

5. T. Rad6, On the problem of Plateau, Ergebnisse der Mathematik und ihrer Grenzgebiete, vol. 2, Springer, 1933. 1948.

6. - Length and area, Amer. Math. Soc. Colloquium Publications, vol. 30,

7. H. Seifert, and W. Threlfall, Lehrbuch der Topologie, Teubner, 1934.

8. L. C. Young, On generalized surfaces of finite topological types, Memoirs of the American Mathematical Society, no. 17, 1955.

Rand Corporation

\section{AN ADDENDUM TO "A NOTE ON SUBSEQUENCES"}

\section{R. CREIGHTON BUCK}

Some years ago, I obtained the following theorem [2]:

THEOREM. A real sequence $\left\{x_{n}\right\}$ is convergent if there exists a regular matrix summability transform $T$ which sums every subsequence of $\left\{x_{n}\right\}$.

In proving this, the initial observation was made: "We may restrict ourselves to bounded sequences." Since then I have received a number of queries relating to this point, and have privately communicated the proof. Together with several other remarks, this is the substance of this note.

Let $\left\{x_{n}\right\}$ be unbounded, and let $T$ be given by a matrix $\left[a_{n k}\right]$. We show that there is a subsequence $\left\{x_{r_{n}}\right\}=\left\{x_{n}^{\prime}\right\}$ which is not summable $T$. In discussing the summability of unbounded sequences, the class of regular transforms falls roughly into two subclasses, those (like triangular methods) that apply to all unbounded sequences, and those that apply only to unbounded sequences that do not increase too rapidly. In the first case, we shall show that any unbounded sequence $x$ has a subsequence $x^{\prime}$ whose transform $T\left(x^{\prime}\right)$ is unbounded (and certainly divergent); in the second case, we produce a subsequence $x^{\prime}$ which is not in the domain of $T$. (Wilansky has informed me that he also has a proof in the case in which $T$ is triangular.)

CASE (i). Let $\left[a_{n, k}\right]$ have infinitely many rows which terminate in a string of zeros (have finite length). If $T$ is regular, then the lengths of these cannot be uniformly bounded. Write $a(n, k)$ for $a_{n, k}$, and choose two strictly increasing sequences $\left\{n_{j}\right\},\left\{k_{j}\right\}$ so that $a\left(n_{j}, k_{j}\right)$ is the last nonzero entry in the $n_{j}$ th row. Since $x=\left\{x_{n}\right\}$ is unbounded,

Received by the editors January 12, 1956. 